\title{
TEMPERAMENTO: ONZE ANOS DE LEVANTAMENTO NO PSYCHOLOGICAL ABSTRACTS
}

\author{
TEMPERAMENT: ELEVEN YEARS OF \\ SEARCHING IN PSYCHOLOGICAL ABSTRACTS
}

\author{
Raquel Souza Lobo GUZZO ${ }^{1}$ \\ Isabel Cristina RIELLO² \\ Ricardo PRIMI ${ }^{3}$ \\ Magali SERRANO ${ }^{4}$ \\ Patrícia do Carmo Pereira ITO ${ }^{5}$ \\ Cristina Coutinho Marques $\mathrm{PINHO}^{6}$
}

\begin{abstract}
RESUMO
Análise da produção científica é um dos pré-requisitos para análise do desenvolvimento científico. Considerando o ressurgimento do temperamento no meio científico nas últimas décadas, este trabalho teve como objetivo verificar o "estado da arte" deste tema. Foi utilizado como suporte bibliográfico o Psychological Abstracts, considerando-se as áreas temáticas: Psicometria, Estatística e Metodologia; Psicologia do Desenvolvimento; Psicologia da Personalidade e Psicologia Educacional. Os dados foram computados em um quadro com indicação do autor, data, volume, número, tema, revista, faixa etária e tipo de artigo (teórico ou pesquisa). Os resultados foram tabulados relacionados à área temática, ao tipo de artigo e à faixa etária. Predominaram no levantamento artigos da área temática Psicologia do Desenvolvimento, artigos do tipo pesquisa e a maioria abordava a faixa etária infantil. Estes resultados confirmam dados da literatura contemporânea que revelam que o temperamento é considerado um dos componentes da personalidade e tem adquirido novas ênfases em Psicologia do Desenvolvimento.
\end{abstract}

Palavras-chave: personalidade, desenvolvimento, busca bibliográfica, produção científica.

\footnotetext{
(1) Professora Doutora em Psicologia do Programa de Pós-Graduação da PUC-Campinas. Endereço para correspondência: Rua: Santa Mônica, 136 - Santa Marcelina - Campinas/SP - Cep: 123094-531. E-mail: rguzzo@mpc.com.br.

(2) Doutora em Psicologia.

(3) Professor Doutor em Psicologia da Universidade São Francisco

(4) Doutoranda em Psicologia na PUC-Campinas e Professora da Unimep.

(5) Doutora em Psicologia.

(6) Doutoranda em Psicologia na PUC-Campinas.
} 


\begin{abstract}
Analysis of scientific production is one of the most important step for analysis of the scientific development. Considering the renaissance of temperament in the scientific way in the last decades, this study had as objective to verify the "state of the art " of this theme. It was used as bibliographical support Psychological Abstracts, being considered the thematic areas: Psychometric, Statistics and Methodology; Development Psychology; Personality Psychology and Education Psychology. The data were computed in a picture with the author's indication, its date, volume, number, theme, journal, age group and article type (theoretical or researches). The results were tabulated related the thematic area, article type and age group. They prevailed in the rising, goods of the area thematic Development Psychology, articles type research and most approached the child's age group. These results confirm data of the contemporary literature that the temperament is considered one of the components of the personality and has been acquiring new emphases in Development Psychology.
\end{abstract}

Key words: personality, development, bibliographical search, scientific production.

O desenvolvimento científico tem sido controverso em sua origem e formas de ler a realidade, ou seja, em suas leis metodológicas. De maneira que a ciência e suas próprias concepções sobre o conhecimento requerem constante análise (Fenati, 1987). Para análise da Ciência, um dos pré-requisitos é que sejam realizados estudos destinados à verificação da produção científica em diferentes áreas de conhecimento e suas respectivas metodologias.

Um tema que tem merecido atenção nas últimas décadas é o temperamento (Rothbart, 1989), o qual tem sido abordado, principalmente, enfocando a avaliação das diferenças individuais, no domínio da personalidade, desenvolvimento e ajustamento social (Goldsmith \& Rieser-Danner, 1990).

Pesquisadores da área têm considerado o temperamento como um dos atributos da pessoa que atua na mediação da interação com o ambiente (Thomas, Chess \& Korn, 1982). Apesar de ser um atributo investigado desde a Grécia Antiga, por Hipócrates e Galeno (Strelau, 1998), permaneceu suprimido do meio científico por um longo período de tempo, ressurgindo em meados de 1950 e 1960 (Rodrigues, 1999). Estudado por diferentes autores, embasados em diferentes abordagens teóricas, o temperamento apresenta diferentes definições, dimensões e instrumentos para sua avaliação (Ito \& Guzzo, 2002).

Considerando a diversidade teórica do construto, uma descrição que contém os fatores essenciais para sua definição e caracterização é oferecida por Allport (1966), o qual estabelece que o "temperamento refere-se aos fenômenos característicos da natureza emocional de um indivíduo, na qual se incluem sua suscetibilidade à estimulação, a intensidade e rapidez usuais de resposta, a qualidade de seu humor predominante, e todas as peculiaridades de flutuação e intensidade de humor, sendo que tais fenômenos são vistos como dependentes da organização constitucional, e portanto, como em grande parte originários da hereditariedade" (p. 57). Complementando esta definição, Clark e Watson (1999) afirmam que as modernas teorias sobre temperamento incorporam a idéia de que o mesmo reflete diferenças individuais de origem biológica, inatas e que fundamentam a personalidade.

Para uma melhor compreensão, clareza na definição e delimitação do construto, são necessários estudos longitudinais, em todas as faixas etárias (Martin \& Wisenbaker, 1995) e ainda o aperfeiçoamento dos métodos e instrumentos de 
avaliação para que os mesmos apresentem qualidades psicométricas adequadas (Angleitner, John \& Lohr, 1986).

Tendo como base os novos e diferentes enfoques sobre temperamento, faz-se necessário que sua produção científica seja conhecida e analisada, para definição de novos estudos, revisões teóricas e metodológicas.

Buscando iniciar uma verificação dos trabalhos desenvolvidos sobre esse tema e suas principais áreas de concentração, a presente pesquisa objetivou investigar artigos sobre temperamento publicados no período de onze anos, analisando sua temática específica, tipo de trabalho e a faixa etária dos participantes.

\section{MÉTODO}

Foi utilizado como suporte bibliográfico o Psychological Abstracts: Nonevaluative Summaries of the Serial and Book Literature in Psychology and Related Disciplines, editado pela American Psychological Association - APA. Sua publicação é mensal e apresenta uma página com orientação detalhada para o leitor, incluindo exemplo de referência dos resumos de artigos, livros e capítulos de livros.

O conteúdo do Psychological Abstracts é apresentado por assuntos divididos em 22 áreas temáticas, desmembradas em 125 subseções. As áreas temáticas são: 1) Psicologia Geral; 2) Psicometria, Estatística e Metodologia; 3) Psicologia Experimental Humana; 4) Psicologia Comparativa e Experimental Animal; 5) Psicologia
Fisiológica e Neurociência; 6) Psicologia e as Humanidades; 7) Sistemas de Comunicação; 8) Psicologia do Desenvolvimento; 9) Processos Sociais; 10) Psicologia Social; 11) Psicologia da Personalidade; 12) Desordens Psicológicas e Físicas; 13) Saúde e Prevenção e Tratamento da Saúde Mental; 14) Psicologia Profissional; 15) Psicologia Educacional; 16) Psicologia Organizacional e Profissional; 17) Psicologia do Esporte; 18) Psicologia Militar; 19) Psicologia do Consumidor; 20) Psicologia Ambiental; 21) Sistemas Inteligentes; e 22) Psicologia Forense e Avaliação Legal.

O construto do temperamento foi pesquisado nos volumes referentes ao período de janeiro de 1985 a junho de 1996, considerando-se apenas os artigos de revistas nas áreas temáticas: Psicometria, Estatística e Metodologia; Psicologia Educacional; Psicologia do Desenvolvimento e Psicologia da Personalidade. Foram selecionados resumos de artigos que apresentavam a palavra-chave "temperamento" no título ou no transcorrer do resumo.

Os dados foram computados em um quadro com indicação de autor, data, volume, número, tema, revista, faixa etária estudada e, se o artigo se referia a um artigo teórico, de revisão da literatura ou de pesquisa, envolvendo o estudo e relação de variáveis.

\section{RESULTADOS}

O levantamento bibliográfico realizado nas quatro áreas temáticas propostas nesta pesquisa resultou num total de 300 artigos publicados

Tabela 1. Freqüência do Tema Temperamento por Áreas Temáticas Pesquisadas.

\begin{tabular}{lcc}
\hline Áreas Temáticas & Freqüência & $\%$ \\
\hline Psicometria, Estatística e Metodologia & 57 & 19,0 \\
Psicologia da Personalidade & 78 & 26,0 \\
Psicologia do Desenvolvimento & 153 & 51,0 \\
Psicologia Educacional & 12 & 4,0 \\
\hline Total & 300 & 100,0 \\
\hline
\end{tabular}


sobre o tema temperamento. Considerando-se a distribuição destes artigos pelas áreas temáticas (Tabela 1), observou-se maior freqüência de artigos na área de Psicologia do Desenvolvimento (51,0\%), seguida de Psicologia da Personalidade (26,0\%). A área temática Psicometria, Estatística e Metodologia concentrou $19,0 \%$ dos artigos publicados, seguida de Psicologia Educacional com 4,0\%. Na análise das categorias por meio do teste de $X^{2}$, para n.g.l. $=3$, n. sig. 0,05 e $X^{2} \tilde{n}=7,81$, foi encontrado $X^{2}{ }_{c}=138,48$, permitindo concluir que foi significativa a concentração de artigos na área de Psicologia do Desenvolvimento $\left(X^{2}=81,12\right)$.

No que diz respeito ao tipo de trabalho (Tabela 2), foram constatados 239 artigos, ou seja, a maioria referia-se a pesquisas realizadas principalmente na área temática de Psicologia do Desenvolvimento (59,4\%). Já os 57 artigos teóricos concentravam-se principalmente na área temática da personalidade $(59,6 \%)$. A análise dos dados considerando o tipo de trabalho, por meio do teste de $X^{2}$, para n.g.l. $=3$, n. sig. 0,05 e $\mathrm{X}_{\mathrm{c}}=7,81$, constatou $\mathrm{X}_{\mathrm{c}}{ }_{\mathrm{c}}=51,92$, o que permite concluir que foi significativa a concentração de trabalhos na categoria artigos de pesquisa.

Considerando a freqüência dos artigos teóricos publicados nas áreas de Psicometria, Estatística e Metodologia, Psicologia da Personalidade e Psicologia do desenvolvimento, a análise dos dados por meio do teste de $X^{2}$, para n.g.l. $=2$, n. sig. 0,05 e $X^{2}{ }_{c}=5,99$ constatou $X^{2}=18,41$, evidenciando que foi significativa a concentração de artigos teóricos na área de Psicologia da Personalidade $\left(X_{c}^{2}=11,84\right)$. Já entre os artigos de pesquisa, $0 X^{2}$, paran.g.I. $=3$, n. sig. 0,05 e $X^{2}=7,81$ constatou $X^{2}{ }_{c}=40,32$, o que permite afirmar que foi significativa a concentração de artigos de pesquisa na área de Psicologia do Desenvolvimento $\left(X_{c}^{2}=28,30\right)$.

Quanto à faixa etária (Tabela 3), nos artigos de pesquisa eram citadas principalmente pesquisas desenvolvidas com crianças $(48,9 \%)$, em menor número apareciam os estudos referentes a adolescentes $(21,8 \%)$ e adultos $(15,1 \%)$. Entre os artigos teóricos, a maioria $(89,4 \%)$ não fazia referência a uma faixa etária específica. $O$ teste de $X^{2}$, para n.g.l. $=6$, n. sig. 0,05 e $X_{c}{ }_{c}=12,6$ constatou $X^{2}{ }_{c}=256,95$, o que permite afirmar que existe uma diferença significativa entre artigos teóricos e de pesquisa, no que diz respeito à faixa etária envolvida nos estudos.

No que diz respeito à distribuição dos artigos de pesquisa considerando-se a faixa etária (Tabela 3), constatou-se que a maioria deles envolvia a participação de crianças $(48,9 \%)$, seguida por adolescentes $(21,8 \%)$ e adultos $(5,4 \%)$, sendo observada nas outras categorias uma freqüência bem menor de sujeitos. A consideração da área temática (tabela 3) evidenciou que em Psicologia do Desenvolvimento predominavam os estudos referentes a crianças (71,1\%), em Psicologia da Personalidade a maioria relacionava-se a participantes adoles-

Tabela 2. Freqüência de Artigos Teóricos e de Pesquisa por Área Temática Pesquisada.

\begin{tabular}{l|cccccc}
\hline \multicolumn{1}{c|}{ Áreas Temáticas } & \multicolumn{2}{c}{ Artigos Teóricos } & Artigos de Pesquisa & \multicolumn{2}{c}{ Não Classificados } \\
\hline & Freqüência & $\%$ & Freqüência & $\%$ & Freqüência & $\%$ \\
\cline { 2 - 7 } Psicometria, Estatística Metodologia & 14 & 24,6 & 42 & 17,6 & 1 & 25,0 \\
Psicologia Personalidade & 34 & 59,6 & 43 & 18,0 & 1 & 25,0 \\
Psicologia Desenvolvimento & 9 & 15,8 & 142 & 59,4 & 2 & 50,0 \\
Psicologia Educacional & 0 & 0,0 & 12 & 5,0 & 0 & 0,0 \\
\hline Total & 57 & 100,0 & 239 & 100,0 & 4 & 100,0 \\
\hline
\end{tabular}


centes $(44,2 \%)$ e adultos (42,0\%). Na área temática Psicometria, Estatística e Metodologia os artigos dividiam-se entre adolescentes $(33,3 \%)$, adultos $(26,2 \%)$ e crianças $(23,8 \%)$. Em Psicologia Educacional predominavam os estudos referentes a adolescentes $(50,0 \%)$ seguidos dos referentes a crianças $(33,4 \%)$.
A análise dos dados, considerando a faixa etária dos sujeitos envolvidos em artigos de pesquisa, por meio do teste de $X^{2}$, para n.g.l. $=5$, n. sig. 0,05 e $X^{2}{ }_{c}=11,1$, constatou $X^{2}{ }_{c}=193,12$, o que permite afirmar que foi significativa a concentração de artigos de pesquisa referentes à participação de crianças $\left(X^{2}{ }_{c}=103,22\right)$.

Tabela 3. Distribuição dos Artigos Teóricos e de Pesquisa por Faixa Etária.

\begin{tabular}{l|cccc}
\hline \multirow{2}{*}{ Faixa Etária } & \multicolumn{2}{c}{ Artigos Teóricos } & \multicolumn{2}{c}{ Artigos de Pesquisa } \\
\hline \multirow{2}{*}{ Criança } & Freqüência & $\%$ & Freqüência & $\%$ \\
\cline { 2 - 5 } Adolescente & 2 & 3,5 & 117 & 48,9 \\
Adulto & 0 & 0,0 & 52 & $1,, 8$ \\
Criança/Adulto & 1 & 1,8 & 36 & 5,4 \\
Criança/Adolescente & 2 & 3,5 & 13 & 2,9 \\
Adolescente/Adulto & 1 & 1,8 & 7 & 4,6 \\
Faixa etária não especificada & 0 & 0,0 & 11 & 0,0 \\
Não classificados & 51 & 89,4 & 0 & 1,3 \\
\hline Total & 0 & 0,0 & 3 & 100,0 \\
\hline
\end{tabular}

Tabela 4. Distribuição dos artigos de Pesquisa por Faixa Etária e Área Temática.

\begin{tabular}{lcccccccc}
\hline \multirow{2}{*}{ Faixa Etária/Áreas } & \multicolumn{2}{c}{$\begin{array}{c}\text { Psicometria, } \\
\text { Estatíst. Metod. }\end{array}$} & \multicolumn{2}{c}{$\begin{array}{c}\text { Psicologia da } \\
\text { Personalidade }\end{array}$} & \multicolumn{2}{c}{$\begin{array}{c}\text { Psicologia do } \\
\text { Desenvolvimento }\end{array}$} & \multicolumn{2}{c}{$\begin{array}{c}\text { Psicologia } \\
\text { Educacional }\end{array}$} \\
\hline & Freq. & $\%$ & Freq. & $\%$ & Freq. & $\%$ & Freq. & $\%$ \\
\cline { 2 - 8 } Criança & 10 & 23,8 & 2 & 4,6 & 101 & 71,1 & 4 & 33,4 \\
Adolescente & 14 & 33,3 & 19 & 44,2 & 13 & 9,2 & 6 & 50,0 \\
Adulto & 11 & 26,2 & 18 & 42,0 & 6 & 4,2 & 1 & 8,3 \\
Criança/Adulto & 0 & 0,0 & 0 & 0,0 & 13 & 9,2 & 0 & 0,00 \\
Criança/Adolescente & 1 & 2,4 & 1 & 2,3 & 4 & 2,8 & 1 & 8,3 \\
Adolescente/Adulto & 4 & 9,5 & 2 & 4,6 & 5 & 3,5 & 0 & 0,0 \\
Não classificados & 2 & 4,8 & 1 & 2,3 & 0 & 0 & 0 & 0,0 \\
\hline Total & 42 & 100,0 & 43 & 100,0 & 142 & 100,0 & 12 & 100,0 \\
\hline
\end{tabular}




\section{DISCUSSÃO}

A freqüência de estudos sobre temperamento nas áreas temáticas selecionadas permite o questionamento de algumas das características dos trabalhos desenvolvidos no período pesquisado. No entanto, devem ser consideradas as limitações encontradas no desenvolvimento do presente levantamento, lembrando que este destinou atenção apenas à palavra-chave "temperamento", seja no título ou no resumo dos trabalhos presentes nas áreas temáticas escolhidas.

Foram encontradas dificuldades quanto à clareza do título dos trabalhos, na definição das palavras-chave e no discurso do resumo. No caso específico do temperamento, existem controvérsias teóricas, sendo que este tema pode ser tratado como sinônimo de personalidade (Eysenck \& Eysenck, 1985).

Por meio da distribuição de freqüência do aparecimento do tema proposto pelas áreas temáticas pesquisadas, observou-se que o maior número de trabalhos concentrava-se na área de Psicologia do Desenvolvimento, aparecendo em seguida a área de Psicologia da Personalidade. Esse dado corresponde ao fato de o temperamento ser considerado como um dos elementos da personalidade (Strelau, 1996), ou tratado como sinônimo dessa (Eysenck \& Eysenck, 1985) ou, ainda, pelo fato de os pioneiros do estudo contemporâneo do temperamento atuarem no domínio da personalidade (Eysenck e Eysenck, 1985) e do desenvolvimento (Thomas, Chess \& Korn, 1982). Segundo Goldsmith e Rieser-Danner (1990), a ênfase atual é nos aspectos do desenvolvimento e na interação de características individuais (temperamento) e ambientais que influenciam a adaptação da pessoa ao seu ambiente.

Aárea Psicometria, Estatística e Metodologia apareceu em terceiro lugar. Discute-se que os avanços no estudo do temperamento só podem ser consistentes quando os instrumentos utilizados garantem a qualidade das avaliações realizadas. Estudos na área da Psicometria são tidos como responsáveis pela melhoria da qualidade das pesquisas em temperamento (Hubert, Wachs, Peters-Martin \& Gandour, 1982). Pode-se dizer que este tipo de pesquisa caminha paralelamente aos avanços teóricos na área, assim como são dependentes deles os estudos transculturais (Angleitner, John \& Löhr, 1986).

A área da Psicologia Educacional concentrou o menor número de publicações. Autores têm tratado da importância de o temperamento ser considerado como uma das fontes de informações para a avaliação educacional (Keogh \& Bess, 1990). Outros têm buscado demonstrar relações do temperamento com estilos de trabalho (Friedensberg, 1985) e com adaptação escolar (Keogh, 1989; Strelau, 1991). Mesmo assim, a investigação do temperamento na área Educacional ainda se encontra em desvantagem em relação às outras áreas aqui constatadas.

A maior freqüência de trabalhos relacionados a artigos de pesquisa pode ser justificada, dentre outros fatores, pelo ressurgimento desse tema no meio científico. Esse ressurgimento requer trabalhos de pesquisa em diferentes áreas de estudo, que possam não só colaborar para a revisão de controvérsias conceituais e metodológicas (Hubert, Wachs, Peters-Martin \& Gandour, 1982), como também levar ao conhecimento de características temperamentais em diferentes situações, ambientes e populações (Strelau, 1991).

Entre os trabalhos de pesquisa, foi realizada uma análise por meio de faixa etária dos participantes envolvidos. A maior freqüência na faixa etária criança corresponde a alguns dados da literatura que apontam para a infância como o maior interesse dos pesquisadores atuais do temperamento (Rothbart, 1989). Principalmente nos EUA existe grande ênfase em estudos de bebês e crianças até a meia infância, sendo utilizado, na maioria dos casos, o relato dos pais ou do adulto responsável como fonte de avaliação dos atributos do comportamento da criança (Goldsmith \& Rieser-Danner, 1990; Martin \& Wisenbaker, 1995).

As faixas etárias adolescentes e adultos apareceram na seqüência. Pode-se dizer que as 
pesquisas nestas faixas etárias não fazem parte da prioridade dos estudos sobre temperamento. No entanto, seria necessário obter maiores informações sobre a região de origem destes trabalhos, já que os EUA têm tradição no estudo do temperamento de crianças (Thomas, Chess \& Korn, 1982) e, por outro lado, na Europa os estudos estendem-se a outras faixas etárias além da infância (Strelau,1991).

Considerando-se separadamente as diferentes áreas pesquisadas de acordo com a faixa etária dos sujeitos, a área Psicologia do Desenvolvimento concentra a maior parte de estudos com crianças, o que reforça a tendência em preocupar-se com as origens biológicas do temperamento por meio de estudos a partir do nascimento (Rothbart, 1989). Psicologia da Personalidade, por outro lado, concentra estudos com adolescentes e adultos e, verificando-se os temas destes trabalhos, observa-se a preocupação por questões adaptativas, stress, impacto social, psicopatologia e profissão.

Em Psicometria, Estatística e Metodologia existe maior distribuição de trabalhos pelas diferentes faixas etárias, fato que pode estar relacionado à necessidade de desenvolvimento e aprimoramento de instrumentos de avaliação destinados a populações diversificadas.

A presente pesquisa, apesar das limitações mencionadas a princípio, ofereceu alguns dados sobre o "estado da arte" no estudo do temperamento nos últimos anos, e também levantou alguns questionamentos específicos quanto: à limitação de estudos sobre temperamento e educação; à necessidade de maiores investigações com faixas etárias diversificadas, além da infância; e às dificuldades de indexação dos estudos realizados com este tema.

\section{REFERÊNCIAS BIBLIOGRÁFICAS}

ALLPORT, W.G. (1966). Personalidade: Padrões e desenvolvimento (D.M. Leite, Trad.). São Paulo: Editora Herder. (Original publicado em 1961).
ANGLEITNER, A., John, O. P. \& Löhr, F.J. (1986). It's what you ask and how you ask it: An itemmetric analysis of personality questionnaires. Em A. Angleitner \& J.S. Wiggins (Orgs.), Personality Assessment via Questionnaires (pp. 61-108). Berlim: Springer-Verla.

CLARK, L. A. \& Watson, D. (1999). Temperament: a new paradigm for trait psychology. Em L.A.Pervin \& O.P. John (Orgs.), Handbook of Personality - Theory and Research (pp. 399-423). New York: Guilford.

EYSENCK, H. \& Eysenck, M.W. (1985). Personality and Individual Differences. London: Plenum Press.

FENATI, R. (1987). A análise das ciências. Síntese, 40 101-105.

FRIENDENSBERG, E. (1985). Reactivity and individual style of work exemplified by constructional type task performance: A developmental study. Em J. Strelau, F.H. Farley \& A. Gale (Orgs.), The Biological Bases of Personality and Behavior: Theories, Measurement and Development (pp.241-253). New York: Hemisphere Publishing Corp/Harper \& Row Publishers.

GOLDSMITH, H.H. \& Rieser-Danner, A. (1990). Assessing early temperament. Em C. R. Reynolds. \& R. W. Kamphaus (Orgs.), Handbook of Psychological and Educational Assessment of Children: Personality, Behavior and Context (pp.245-271). New York: Guilford Press.

HUBERT, N.C., Wachs, T., Peters-Martin, P. \& Gandour, M.J. (1982). The study of early temperament: Measurement and conceptual issues. Child Development,53.571 - 600.

ITO, P. C. P. \& Guzzo, R. S. L. (2002). Diferenças Individuais: temperamento e personalidade; importância da teoria. Estudos de Psicologia, 19 (1), 91-100.

KEOGH, B.K. (1989). Applying temperament research to school. Em G.A. Kohnstamm, J.E. Bates \& M. K. Rothbart (Orgs.), Temperament in Childhood (pp.437-450). Chilchester: John Wiley \& Sons. 
KEOGH, B.K. \& Bess, C.R. (1990). Assessing temperament. Em H.L. Swanson (Org.), Handbook of Learning Abilities: Theory, Research and Practice (pp.13-329). Texas: Pro Ed.

MARTIN, R.P. \& Wisenbaker, J. (1995). Prediction of teacher rated behavior problems at 12-years from maternal ratings of temperament in infancy. Paper presented at the $7^{\text {th }}$ Meeting of the International Society for the Study of Individual Differences. Warsaw, Poland.

RODRIGUES, M.M.P. (1999). Desenvolvimento das relações pais - filhos: uma abordagem evolutiva. Em A. M. Carvalho (Org.), O mundo social da criança: natureza e cultura em ação (pp. 53-70). São Paulo: Casa do Psicólogo.

ROTHBART, M.K. (1989) Temperament and Development. Em G. A. Kohstamm, J. E. Bates \& M. K. Rothbart, M.K. (Orgs.),
Temperament in Childhood (pp.187-247). Chilchester: John Wiley \& Sons.

STRELAU, J. (1991). Temperament and giftedness in children and adolescents. Paper presented at the $9^{\text {th }}$ World Conference on Gifted and Talented Children - The Haque, Netherlands.

STRELAU, J. (1996). The regulative theory of temperament: current status. Personality and Individual Differences,20 (2), 131-142.

STRELAU, J. (1998). Temperament: a psychological perspective. New York: Plenum Press.

THOMAS, A., Chess, S. \& Korn, S.J. (1982) The reality of difficult temperament. Merril-Palmer Quarterly, 28 (1), 1-20.

Recebido para publicação em 06 de maio de 2003 e aceito 01 de dezembro de 2003. 